\title{
Intonation in neurogenic foreign accent syndrome
}

\author{
Anja Kuschmann ${ }^{\mathrm{a}}$, Anja Lowit ${ }^{\mathrm{a}}$, Nick Miller ${ }^{\mathrm{b}}$, Ineke Mennen $^{\mathrm{c}}$ \\ ${ }^{a}$ Strathclyde University Glasgow, School of Psychological Sciences and Health, Division of Speech and \\ Language Therapy, Sir Henry Wood Building, 76 Southbrae Drive, Glasgow G13 1PP, United Kingdom \\ ${ }^{b}$ University of Newcastle Upon Tyne, Institute of Health and Society, Speech Language Sciences, George VI \\ Building, Newcastle Upon Tyne NE1 7RU, United Kingdom \\ ${ }^{c}$ Bangor University, School of Linguistics and English Language, Brigantia Building, Gwynedd LL57 2DG, \\ United Kingdom
}

\begin{abstract}
Foreign accent syndrome (FAS) is a motor speech disorder in which changes to segmental as well as suprasegmental aspects lead to the perception of a foreign accent in speech. This paper focuses on one suprasegmental aspect, namely that of intonation. It provides an in-depth analysis of the intonation system of four speakers with FAS with the aim of establishing the intonational changes that have taken place as well as their underlying origin. Using the autosegmental-metrical framework of intonational analysis, four different levels of intonation, i.e. inventory, distribution, realisation and function, were examined in short sentences. Results revealed that the speakers with FAS had the same structural inventory at their disposal as the control speakers, but that they differed from the latter in relation to the distribution, implementation and functional use of their inventory. The current results suggest that these intonational changes cannot be entirely attributed to an underlying intonation deficit but reflect secondary manifestations of physiological constraints affecting speech support systems and compensatory strategies. These findings have implications for the debate surrounding intonational deficits in FAS, advocating a reconsideration of current assumptions regarding the underlying nature of intonation impairment in FAS.
\end{abstract}

Keywords: foreign accent syndrome, intonation, autosegmental-metrical, IViE 


\section{Introduction}

Foreign accent syndrome (FAS) can be defined as a motor speech disorder that is characterised by changes to segmental and suprasegmental speech aspects leading to the emergence of a perceived foreign accent in speech. That is, listeners of the same language community perceive the speech of the individual as that of a non-native speaker, or alternatively, that of a native speaker from a different dialectal community. Despite the speech impairments speakers remain largely intelligible and relatively fluent.

The term foreign accent syndrome was originally coined by Whitaker (1982) to refer to speakers presenting with an acquired change in accent after brain damage. According to this definition, the change in accent is related to a neurological incident, i.e. damage of the central nervous system and excludes the possibility that psychogenic aetiologies might have a role in the altered speech patterns. The assumption that FAS should be neurogenic and not psychiatric in nature was maintained by Blumstein and Kurowski (2006). However, recent case studies suggest that structural damage to the brain may not necessarily be a prerequisite for a speaker to acquire a foreign accent, and that the observed changes can at least partially be attributed to psychogenic causes. This has been reflected in the new taxonomy suggested by Verhoeven and Mariën (2010). Reported cases of psychogenic origin of FAS include episodes of psychotic schizophrenia (Reeves, Burke \& Parker, 2007), bipolar disorder (Poulin, Macoir, Paquet, Fossard \& Gagnon, 2007), and conversion disorder (Haley, Roth, Helm-Estabrooks \& Thiessen, 2010; Verhoeven, Mariën, Engelborghs, D’Haenen \& De Deyn, 2005).

Despite this recent increase in the number of reported psychogenic cases the majority of FAS cases documented in the literature concern adult patients who present with a wide variety of 
neurological aetiologies. In most reported cases, the emergence of the foreign accent in speech is the result of structural brain damage, predominantly vascular brain lesions. Often, but not exclusively, these affect the cortical motor speech regions of the left hemisphere, including the prerolandic motor cortex, the frontal motor association cortex or the striatum (Kurowski, Blumstein \& Alexander, 1996; Mariën et al., 2006; Mariën, Verhoeven, Wackenier \& Engelborghs, 2009).

The majority of case descriptions in the literature acknowledge the relevance of segmental as well as suprasegmental speech characteristics in defining FAS. At the segmental level, the production of vowels and consonants is affected. Frequently reported vocalic errors include alterations in vowel length, i.e. lengthening (Blumstein, Alexander, Ryalls, Katz \& Dworetzky, 1987; Graff-Radford, Cooper, Colsher \& Damasio, 1986) as well as shortening (Ingram, McCormack \& Kennedy, 1992; Perkins, Ryalls, Carson \& Whiteside, 2010) and tenseness (e.g. Katz, Garst \& Levitt, 2008; Whitaker, 1982). Commonly reported consonantal errors include changes in manner and place of articulation (Ardila, Rosselli \& Ardila, 1988; Verhoeven \& Mariën, 2010), voicing/devoicing of plosives and fricatives (Gurd, Bessell, Bladon \& Bamford, 1988; Scott, Clegg, Rudge \& Burgess, 2006) and hyper- or hypoaspiration of plosives (Kurowski et al., 1996; Moen, 2006).

Suprasegmental alterations frequently affect stress, rhythm and intonation. Regarding stress, misplacement of lexical stress has been reported (Scott et al., 2006; Whitaker, 1982), as well as a tendency towards equalising stress across syllables (Berthier, Ruiz, Massone, Starkstein \& Leiguarda, 1991; Blumstein et al., 1987). Rhythmic alterations appear to be the result of changes to the syllabic structure of words caused by schwa-insertions and a restricted ability to reduce vowels in unstressed syllables (e.g. Blumstein et al., 1987; Katz et al., 2008; 
Monrad-Krohn, 1947). Reported changes in intonation include a variety of - sometimes contradictory - features: a higher mean pitch (e.g. Blumstein et al., 1987; Coelho \& Robb, 2001; Ryalls \& Whiteside, 2006); inappropriately large and sharp pitch excursions on prominent syllables (e.g. Avila, González, Parcet \& Belloch, 2004; Moonis et al., 1996); exaggerated terminal falls (Ingram et al., 1992; Moen, 2006); reduced pitch range (GraffRadford et al., 1986; Kanjee, Watter, Sévigny \& Humphreys, 2010; Verhoeven \& Mariën, 2010); and the inappropriate use of intonation to indicate statements (i.e. use of sharp rises where falls would be expected, e.g. Berthier et al., 1991; Blumstein et al., 1987; MonradKrohn, 1947) or questions (i.e. use of falls where rises would be expected, e.g. Graff-Radford et al., 1986; Moen, 2006).

Studies investigating the speech patterns in FAS have frequently identified an underlying disturbance in speech prosody, i.e. rhythm and intonation, as a main factor contributing to the symptom complex of FAS and the perceived foreign accent (e.g. Blumstein et al., 1987; Kurowski et al., 1996). However, whilst segmental features are usually described in great detail using a sizeable data set, detailed experimental investigations and formal descriptions of intonation or other prosodic aspects are rare. The studies that have looked into aspects of intonation have largely relied on perceptual judgements and broad acoustic measurements. As a result, studies frequently established the presence of changes in this component but did not provide the necessary detail to be able to draw conclusions as to the exact manifestation of the intonational changes and their potential underlying nature. It is thus unclear whether the changes at the level of intonation reflect features of a core impairment or compensatory strategies to deal with impairment in another speech aspect. However, distinguishing between both types of features has the potential to determine the intonational aspects that are indeed compromised in FAS (Miller, Lowit \& O'Sullivan, 2006). There is thus a gap in the literature 
between the recognised relevance of intonational changes in defining FAS on the one hand, and the efforts undertaken to determine the underlying nature of these alterations on the other.

This study works towards filling this gap by providing a systematic linguistic account of the internal organisation of the intonation system in FAS. The aim is to provide insights into the underlying nature of intonation deficits in FAS by identifying the level of intonation impairment and by differentiating between core impairments and potential compensatory strategies.

\section{Method}

\subsection{Participants}

Four individuals with FAS and four age-, gender- and dialect-matched control speakers participated in the study (table 1). All participants were monolingual speakers of English. Informal assessment ensured that they had no uncorrected visual or auditory impairment, no signs of depression and no history of speech and language difficulties. In all four speakers with FAS the neurogenic origin of the foreign accent was established by relevant health care professionals. However, the possibility of a psychogenic component contributing to the observed speech changes could not be entirely excluded.

Table 1 about here

\subsection{Materials}

Participants performed a number of production tasks designed to gather baseline and phonetic speech measures (speech rate, pitch range, maximum performance tests; see below), and to 
specifically investigate intonation (sentence reading task). The sentence reading task formed part of a larger study on FAS that elicited scripted as well as unscripted speech data including a reading passage, a picture description and a monologue (Kuschmann, 2011).

The sentence reading task was specifically designed to examine the realisation of intonation in FAS in relation to the linguistic function of information status, i.e. the signalling of new and given information. New information refers to the informative part of an utterance, whereas given information represents the part of the utterance that can be inferred from the preceding context (Halliday, 1967). The pragmatic role of elements in the ongoing discourse is essential to effective communication as it contributes to the structuring of information by directing the listener's attention to the relevant information of the utterance. In West Germanic languages including English, information status is signalled in a binary, categorical fashion: new information is routinely assigned a pitch accent, whereas given information (in post-focal position) is expected to undergo de-accentuation (e.g. Baumann, 2006; Chafe, 1994; Cruttenden, 2006; Gussenhoven, 2004). The term pitch accent - as it is used in this study - denotes a phonological feature that serves as a perceptual cue to prominence in intonation languages (Ladd, 1996). As a consequence, this definition does not cover word level phonemic differences that exist in languages such as Swedish or Norwegian.

In order to investigate information status, a set of ten sentences was devised. The sentences were controlled for length, syntactic structure, word stress patterns as well as sonorance to facilitate pitch track analysis:

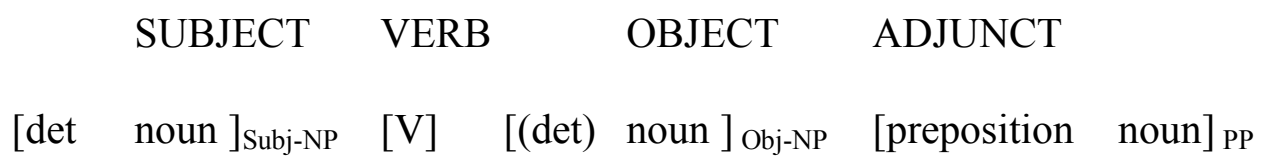


[The $][$ gardener $]$ [grew $]$ [roses $\quad[$ in $]$ [London]

The words in the subject, object and adjunct position of each sentence formed the target words for the investigation of information status. Specifically, two factors were relevant: information status (new vs. given) and sentence position (initial vs. medial vs. final), resulting in four different conditions. In the baseline condition, all three target words were contextually new, whereas in the remaining three conditions the position of the new information varied between initial, medial and final position. Overall, each speaker produced 40 sentences containing 120 target words, of which 60 were new and 60 were given. After excluding unanalysable sentences, a total of 276 sentences and 828 target words were analysed for the eight speakers, of which 416 were new and 412 were given.

Sentences were elicited through a question-answer paradigm, with questions varying according to the target constituent. The sentences, which were randomised and separated by fillers, were presented with a combination of auditory and visual prompts to maximise correct processing of the required linguistic structures. The auditory prompts were provided by a male and a female speaker of Standard Southern British English, who were instructed to speak in a natural way and at a normal speech rate. Each question prompt was followed by a visual presentation of the answer on the same slide. In order to facilitate focus on the correct item, target words were underlined. After completing four practice items, participants worked through the slides at their own pace. In case of slips of the tongue, word omissions or hesitations, they were asked to repeat the sentence.

\subsection{Recording procedure}


Participants were recorded in a quiet room at home or at a nearby university. Recordings were made using a portable DAT-recorder (TASCAM DA-P1) and a condenser microphone (Beyerdynamic MPC 65 V SW). The speech recordings were subsequently converted to audio files in .wav format using Kay Elemetrics Multispeech System, at a sampling rate of 44.1 kHz. The analysis of the speech data was conducted using Praat speech analysis software (version 5.0.11 (C) Boersma \& Weenink, 1992-2011). The fundamental frequency contour was checked to detect halving and doubling errors of the pitch tracker which, if necessary, were corrected by hand.

\subsection{Data annotation and analysis}

\subsubsection{Baseline and phonetic measures}

Three Maximum Performance Tests (MPTs) were conducted to assess phonatory and pulmonary resources. Maximum phonation duration (MPD) provided information on the respiratory support that was available to sustain phonation (in seconds); a loudness glide established the abilities of volume control (in decibels) and pitch modulation was assessed by means of a pitch glide (in Hertz). Each of the tasks was demonstrated once, before the participants were asked to repeat each test twice. The mean of both performances was used for further analysis.

Speech rate was calculated for the reading passage, the picture description and the monologue by dividing the total number of syllables per sample by the overall speaking time including pauses and hesitations. 
Pitch range measures of level and span (Ladd, 1996) were carried out on each sentence utterance of the sentence set. Level refers to the overall pitch height used by a person during speech, and span to the variation of frequencies that is covered by a speaker's voice.

For level the value of the final low (FL) was taken; for span the F0 value of the final low (FL) was deducted from the highest value measured on any of the target words (mostly H1). In order to account for gender differences in pitch height, the $\mathrm{Hz}$ values were converted into semitones (ST) (Mennen et al., 2008; Nolan, 2003; Reetz, 1999).

\subsubsection{Intonation measures}

The intonation data were analysed within the autosegmental-metrical (AM) framework of intonational analysis (Pierrehumbert, 1980; for a comprehensive overview see Ladd, 1996). The AM framework is an explicitly phonological approach to analysing intonation that characterises intonational structures independently from the phonetic features of the tune structure, i.e. the sequence of tones. The phonological categories of intonation are analysed in terms of sequences of $\mathrm{H}(\mathrm{igh})$ and L(ow) target tones, which are further organised into pitch accents and boundary tones depending on their respective association with stressed syllables or phrase boundaries. The data were annotated using an adaptation of the Intonational Variation in English (IViE) transcription system (Grabe, Post \& Nolan 2001). IViE is one of many transcription systems based on the AM framework, and was specifically developed for intonational analyses of different dialects in the British Isles, thus allowing the use of one annotation system for all our participants. Six levels of annotation were used: 1) a syllable-bysyllable orthographic transcription, 2) a transcription indicating phrase boundaries and prominent syllables, 3) a phonetic transcription of pitch movements around the prominent syllables, 4) a phonological transcription of pitch accents and boundary tones, 5) a transcription of the information status and 6) comments. For the phonological transcription, 
the following labels were employed: $\mathrm{H}^{*}$ (high pitch accent), $\mathrm{L}^{*}$ (low pitch accent), $\mathrm{H}^{*} \mathrm{~L}$ (falling pitch accent), ! H*L (downstepped pitch accent), $\mathrm{L}^{*} \mathrm{H}$ (rising pitch accent), $\mathrm{L} * \mathrm{HL}$ (rise-fall pitch accent) and $\mathrm{H}^{*} \mathrm{LH}$ (fall-rise pitch accent). In addition to the different pitch accent types, the following boundary tone labels were used: \% $\%$ and L\% (phrase-initial and final low boundary tone), $\% \mathrm{H}$ and $\mathrm{H} \%$ (phrase-initial and -final high boundary tone) and \% (phrase-final level boundary tone).

Based on the annotation of the data, and following Mennen, Schaeffler, Watt and Miller (2008), a variety of measures pertaining to four dimensions of intonation were conducted, i.e. the inventory, distribution, implementation and function of the different categorical elements of intonation. According to Ladd (1996) these four dimensions comprehensively describe the intonation system of a language, a language variety or dialect. The inventorial analysis established which pitch accents and boundary tones the individuals with FAS had at their disposal, whereas the distributional analysis concerned the frequency of use of these elements. The analysis of the implementation patterns involved the assessment of accentuation and phrasing by measuring the pitch-accent-syllable ratio, i.e. the overall frequency of pitch accentuation, and the mean length of intonation phrases. For the analysis of function, the use of pitch accents to indicate new and given information was evaluated.

Intra- and inter-rater reliability for transcription was completed on $10 \%$ of the data for intonation phrase boundaries, prominent syllables and classification of the structural elements, i.e. pitch accents and boundary tones. Reliability for intra-rater transcription was consistently over 95\% indicating a high degree of agreement (IP boundaries: 96.2\%; prominent syllables: 97.8\%, phonological labels: 95.2\%). Inter-rater agreement was high for IP boundaries (95.6\%) and prominent syllables (88.4\%). The congruence pertaining to the labelling of the 
structural elements was $81.2 \%$, reflecting good agreement for pitch accents and boundary tones, and matching or exceeding reliability scores usually obtained for intonational transcriptions (Pitrelli, Beckman \& Hirschberg, 1994).

\section{Results}

\subsection{Baseline and phonetic measures}

Table 2 provides a summary of the mean values of maximum performance, speech rate and pitch range per speaker and speaker group.

Statistical analyses (Mann-Whitney U test) of the MPTs show significantly shorter maximum phonation duration times $(\mathrm{p}=0.05)$ and less change in $\mathrm{F} 0$ for the pitch glide $(\mathrm{p}=0.05)$ for the speakers with FAS compared to the healthy control speakers, but no statistically significant difference for loudness $(\mathrm{p}=0.827)$. Results for speech rate revealed a significantly slower speech rate for the speakers with FAS compared to the control group for the reading passage $(p=0.021)$, but not for the picture description $(p=0.245)$ or the monologue task $(p=0.243)$. In contrast to the pitch glide analysis, the analysis of pitch level and span indicated a significantly higher level and wider pitch span for the speakers with FAS than for the control participants (level: $\mathrm{p}<0.001$, span: $\mathrm{p}<0.001$ ).

Table 2 about here

\subsection{Dimensions of intonation}

Due to the relatively small number of participants descriptive statistics were considered most appropriate to present the findings pertaining to the different dimensions of intonation. The strong similarities observed across speakers' performances allowed the pooling of data by 
group for result presentation. Where individuals deviated noticeably from the group norm, this is highlighted in the discussion below.

\subsubsection{Inventory}

Figure 1 displays the different types of pitch accents used by the FAS group and the control group. Both speaker groups used the pitch accents $\mathrm{L}^{*}, \mathrm{H}^{*}, \mathrm{H}^{*} \mathrm{~L}, ! \mathrm{H}^{*} \mathrm{~L}$ as well as $\mathrm{L}^{*} \mathrm{H}$. The only difference between the two groups could be observed in the use of the complex pitch accent $\mathrm{L} * \mathrm{HL}$, which only featured in the inventory of the FAS group. With regard to boundary tones both speaker groups were found to employ low, high as well as level boundary tones (cf. figure 2). Overall, the results of the inventorial analysis revealed a similarly rich inventory of pitch accents and boundary tones for both speaker groups, suggesting that the speakers with FAS had the same intonational patterns at their disposal as the healthy control speakers.

Figure 1 about here

Figure 2 about here

\subsubsection{Distribution}

The distributional analysis of the pitch accent inventory indicates that both speaker groups displayed very similar patterns. The falling pitch accent $\mathrm{H}^{*} \mathrm{~L}$ represented the most frequently used pitch accent followed by the high tone $\mathrm{H}^{*}$, whilst pitch accents $\mathrm{L}^{*}$ and $\mathrm{L}^{*} \mathrm{HL}$ were only rarely employed (cf. figure 1). Differences between speaker groups occurred in relation to the use of the rising pitch accent $\mathrm{L} * \mathrm{H}$, which represented more than $10 \%$ of the pitch accents used by the FAS group, but was only marginally employed by the control group. However, individual speaker analyses revealed that the increased use of $\mathrm{L}^{*} \mathrm{H}$ in the speakers with FAS 
could be directly attributed to FAS2, who employed this tone in $43 \%$ of all occasions. In the remaining speakers with FAS the falling pitch accent $\mathrm{H}^{*} \mathrm{~L}$ prevailed. The higher prevalence of $\mathrm{L}^{*} \mathrm{H}$ in the FAS data is thus the result of speaker-specific preference.

The distribution of boundary tones showed that the beginning of intonation phrases was primarily marked using low level tone $\% \mathrm{~L}$, whereas the level tone $\%$ represented the most prevalent tone to mark the end of intonation phrases in both speaker groups (cf. figure 2). Clear differences were observed regarding the use of the high boundary tone to mark the end of phrases. Whilst the control group used this tone only occasionally, it accounted for $26 \%$ of the phrase-final boundary tones in the FAS group (cf. table 3). Individual speaker analyses showed that the higher percentage of $\mathrm{H} \%$ in the FAS group was found across all four speakers with FAS, with its use ranging from $16 \%$ to $36 \%$ of boundary tones. The analysis further revealed that the high boundary tones were exclusively used to demarcate sentence-internal as opposed to sentence-final phrase boundaries.

Table 3 about here

\subsubsection{Implementation in terms of phrasing and accentuation}

Table 4 presents the results of the phrasing as well as accentuation patterns, which were established by means of mean length of intonation phrase (IP) and pitch-accent-syllable ratio. Regarding the phrasal behaviour, the analysis of the mean IP length revealed that the control speakers had an average IP length of at least nine syllables, producing the majority of sentences as one IP. By contrast, the IPs produced by the speakers with FAS were on average three syllables shorter. There was some variability between FAS speakers regarding the extent to which IPs were shortened, i.e. FAS1 and FAS4 produced IPs half the length of those of the 
control speakers, FAS2 and FAS3 produced phrases that were only about one third shorter (cf. table 4).

In relation to the frequency of pitch accentuation, the analysis of the pitch accent-syllable ratio revealed that the control speakers produced on average one pitch accent for every four syllables, whereas the speakers with FAS did so for every three syllables, resulting in a higher frequency of pitch accented words than the control speakers. Further analysis showed that the higher frequency of pitch accents in FAS was partly due to the low frequency of deaccentuation of target words (cf. 3.2.4) and partly to the fact that speakers assigned pitch accents to other words, i.e. the verbs, determiners and prepositions.

Table 4 about here

\subsubsection{Function of intonation: the marking of information status}

Figure 3 displays the percentage of pitch accentuation for new and post-focally given information per speaker. New items were consistently assigned a pitch accent by both control speakers and speakers with FAS suggesting that the marking of new items in discourse followed similar patterns in both speaker groups. However, considerable differences became apparent in relation to the marking of given information. The control speakers produced pitch accents on $7 \%$ to $30 \%$ of given items, which shows that de-accentuation was the preferred option in this speaker group. The analysis of the speakers with FAS revealed a considerably different pattern with accentuation rates that consistently ranged above $70 \%$. This finding suggests that the speakers with FAS predominantly accentuated given items in post-focal position, with de-accentuation constituting the less frequent pattern.

Figure 3 about here 


\section{Discussion}

The purpose of this study was to systematically describe the internal organisation of the intonation system in FAS along its four dimensions in an attempt to identify the nature of intonation impairment in FAS. The dimensional analysis revealed that the speakers with FAS had a similarly rich inventory of pitch accents and boundary tones at their disposal as the control speakers, but differed in terms of the distribution, implementation and functional use of these categories.

Regarding the inventory of structural categories both speaker groups used the same categories. This suggests that the speakers with FAS were able to employ the same structural categories as the healthy control speakers, and thus that the abstract phonological representations of pitch accents and boundary tones were retained in this group. A similar observation was made by Verhoeven and Mariën (2002, 2004, 2010), who investigated the intonation patterns of a Dutch speaker with FAS. They found that their speaker successfully produced the four major Dutch intonation contours, adhering to rules of intonational wellformedness, and concluded that intonation contours in that speaker were unaffected.

The analysis of the distributional patterns revealed differences between speaker groups concerning the use of the rising pitch accent $\mathrm{L} * \mathrm{H}$ and the high boundary tone $\mathrm{H} \%$, which were more frequently employed among the speakers with FAS. While the higher prevalence of $\mathrm{L}^{*} \mathrm{H}$ was the result of speaker-specific performance, the higher incidence of $\mathrm{H} \%$ could be observed in all speakers with FAS. Importantly though, this boundary tone was only employed to demarcate sentence-internal phrase boundaries, whereas sentence-final boundaries were marked by a low level tone. A phrase-final rise in intonation is commonly 
used by speakers as a marker of continuation. Given the frequent division of sentences into smaller phrasing units, the speakers with FAS may have employed $\mathrm{H} \%$ at the end of sentenceinternal phrase boundaries to signal that the sentence was not finished yet. This suggests that the FAS group had sufficient control over their intonation realisation to mark these internal boundaries differently to sentence-final boundaries. In this light the more frequent use of $\mathrm{H} \%$ in FAS may be interpreted as a deliberate strategy employed to signal that there was more information to come, rendering the observed changes at this level compensatory in nature.

The interpretation of the use of $\mathrm{H} \%$ as continuation marker in FAS is again supported by findings from Verhoeven and Mariën $(2002,2004,2010)$, who observed a particularly frequent use of continuation contours in the conversational data of their Dutch speaker with FAS. The authors argue that the speaker might have used these contours to indicate that there was still information to come, which may not always be clear due to a slow speech rate. Slower than normal speech rate has frequently been observed in FAS (Ardila et al., 1988; Avila et al., 2004; Berthier et al., 1991; Blumstein et al., 1987; Kurowski, et al., 1996) and was also present in the current speakers with FAS. The parallels between the current speakers with FAS and Verhoeven and Mariën's $(2002,2004,2010)$ Dutch speaker suggest that the phrase-final rising intonation patterns may have been used for similar pragmatic reasons. In light of these findings it would also be worth re-investigating the data of a number of previous FAS case studies that reported phrase-final rising F0 contours where a fall would have been expected (e.g. Berthier et al., 1991; Blumstein et al., 1987; Katz et al., 2008; Monrad-Krohn, 1947; Moonis et al., 1996) in order to explore whether these rises could also have represented continuation markers. 
In terms of the structural implementation, the speakers with FAS produced a greater number of pitch accents than the control participants, combined with shorter intonation phrases. Both findings are consistent with results from previous FAS reports. Wendt, Bose, Scheich \& Ackermann (2007) observed twice the number of pitch accents in a German speaker with FAS than the matched control speaker, as well as significantly shorter phrasing units. A higher rate of pitch accentuation was also reported by Berthier et al. (1991) and Graff-Radford et al. (1986). Both studies reported that in some sentences the speakers with FAS accented almost every content word.

The higher rate of accentuation in FAS was reflected in the results of the marking of information status using intonation. Whilst control speakers primarily de-accented given information, in the speakers with FAS accentuation prevailed. This finding suggests that the phonological marking of information status in the current speakers with FAS was compromised.

A possible reason for the observed changes at the implementation level were issues with phonatory and respiratory support as evidenced in the restrictions observed in some of the maximum performance tests. The current speakers with FAS had reduced breath support, which is likely to have triggered the division of utterances into smaller phrasing units. This, in turn, could have resulted in a higher frequency of accentuation, as every intonation phrase has to bear at least one pitch accent (Pierrehumbert, 1980). An increased number of given items thus had to be assigned a pitch accent in order to adhere to the principles of intonational wellformedness. On this basis, the lack of de-accentuation observed in the speakers with FAS could be interpreted as less of a core deficit in discriminating between new and given information than a clash of the de-accentuation constraint with universal rules of intonational well-formedness. The observed difficulties in marking given information by means of de- 
accentuation could thus at least partly be interpreted as a consequence of the structural changes seen at the level of prosodic phrasing.

However, this explanation cannot account for all the cases where speakers with FAS failed to de-accent in post-focal positions as $51 \%$ of sentences were realised as one IP, but target words were still not always de-accented. Similar behaviour was evident in the CON group, but to a lesser extent ( $9 \%$ of sentences), indication that de-accentuation might be the expected pattern for postfocal given information, but is clearly not the only option available to speakers (Cruttenden, 2006; Prince, 1981; Terken \& Hirschberg, 1994). However, this does not explain the considerably higher incidence of this behaviour in the FAS group. The question therefore arises whether the observed pattern might after all be the result of a core impairment of intonation, or could be explained by other physiological deficits beyond breath support. The current data suggest that the presence of a primary intonation deficit is the less likely of the two options, given that the speakers with FAS showed no differences in the structural inventory of pitch accents and appeared to be able to use intonation in a compensatory way at the distributional level. In addition, although they showed a reduced range in the pitch glide task, their performance in connected speech was at least comparable to that of the control speakers, as reflected by a higher span. Discrepancies in performance between speech and non-speech tasks have been well documented in the literature (e.g. Ziegler, 2002) and were exacerbated in the current sample by the highly variable results for the span measure. There is thus no clear indication that physiological restrictions were the cause of the higher incidence of pitch accentuation in post-focal position observed in the FAS group. One interesting observation is though that those FAS speakers with higher span levels were also the ones who produced the greatest number of pitch accents per IP (cf. tables $2 \& 3$ ). Although this finding was not replicated in the control group where speakers were relatively stable in the pitch 
accent ratio but highly variable in their span, these results could suggest that there is a link not only between respiratory support and the number of pitch accents produced, but also their phonetic realisation.

Given that F0 performance, as assessed in this study, could clearly not fully explain the higher number of pitch accents in the current FAS groups, another plausible account rests on possible problems in the coordination of respiration and phonation at a level above mere weakness or loss of muscle tone. These are common in apraxia of speech (Kent \& Rosenbek, 1982, 1983). Although there was no clear indication of the presence of apraxia in the current FAS group, this possibility should be considered nonetheless, given the reported association of FAS with features of apraxia of speech (Ackermann, Hertrich, \& Ziegler, 1993; Coelho \& Robb, 2001; Mariën et al., 2006, 2009; Miller et al., 2006; Varley \& Whiteside, 2001; Whiteside \& Varley, 1998).

Further articulatory factors that might have affected the implementation of intonation patterns are speech rate and effort. The effects of slower rate on other prosodic components are not very well understood but it is not unfeasible that prolonged syllable durations could have affected accent placement, given that pitch accents are associated with phonetically salient syllables. Increased articulatory effort could be a further explanation, as this might have resulted in greater excursions of F0 modulation irrespective of linguistic function.

In summary, the findings of the present study suggest that underlying physiological changes affecting areas such as breath support, coordination and articulatory effort could be the main cause of the observed intonational alterations seen at the levels of distribution, implementation and function in FAS. Rather than dealing with an underlying disturbance of intonation and prosody, as suggested by earlier studies (e.g. Blumstein et al., 1987) the observed changes more likely represent secondary deficits or reflect compensatory 
mechanisms adopted to deal with the primary speech impairment. Support for the assumption of affected motor control of speech movements comes from a study by Fridriksson et al. (2005). Combining structural and functional brain imaging the authors suggest that their case of FAS represents a compensatory response to impaired motor regulation of speech.

As a result of these findings, a model was devised (figure 4) that attempts to express the relationship between the different aspects of intonation production in order to pinpoint the locus of breakdown of intonational realisation as hypothesised for the current FAS group. It should be noted that the suggested structure only accounts for the results of the present study and may have to be redefined and elaborated when more evidence on the intonational impairment in FAS becomes available. For instance, there is a potential for a timing and F0 dissociation as evidenced in other disorders such as non-fluent aphasia (Danly \& Shapiro, 1982; Ryalls, 1982). Future studies will have to establish whether this might also be the case for FAS.

Figure 4 about here

The proposed model assumes a direct relationship between the abstract phonological representations, i.e. pitch accents and boundary tones, and their implementation in terms of accentuation and phrasing, which are connected via a unidirectional link. Whilst both phrasing and accentuation are directly associated with the phonetic means of implementation, the connection of both aspects to the function of intonation in marking information status is less straightforward. Here, the present data only suggest a direct link to accentuation, whereas phrasing only indirectly has an impact on the marking of information structure, with accentuation serving a mediating role. In addition, the findings of the present study suggest 
that physiological restrictions and/or coordination issues might have had an impact on a speaker's performance, with phrasing of utterances as well as the phonetic attributes in FAS speech being influenced by these factors.

\section{Conclusions}

The detailed account of the intonation component in speakers with FAS using a wellestablished linguistic approach to analyse intonation revealed a retained inventory of abstract intonational categories in FAS combined with difficulties in realising these abstract representations. As a consequence, speakers with FAS may not have effectively indicated information status by means of intonation. However, their intonation contours were wellformed throughout. This fact suggests that the speakers with FAS seemed to be aware of the intonational rules and were able to successfully deploy and adjust them within a system that is constrained by physiological impairments. Although further investigations are necessary to pinpoint the exact level of breakdown and the relationship between the various levels, the current analysis of the intonation system in FAS has demonstrated that the use of an explicitly linguistic framework clearly has the potential to take the debate surrounding intonation in FAS further, advocating the reconsideration and possibly redefinition of current assumptions of intonation impairment in FAS.

\section{Acknowledgements}

We gratefully acknowledge partial support for this project from the British Academy through research grant SG-44232. The final author was supported the ESRC grant RES-000-22-2419. 


\section{References}

Ackermann, H., Hertrich, I. \& Ziegler, W. (1993). Prosodische Störungen bei neurologischen Erkrankungen - Eine Literaturübersicht. Fortschritte der Neurologie - Psychiatrie, 61, 241-253.

Ardila, A., Rosselli, M. \& Ardila, O. (1988). Foreign accent: an aphasic epiphenomenon? Aphasiology, 2(5), 493-499.

Avila, C., González, J., Parcet, M.-A. \& Belloch, V. (2004). Selective alteration of native, but not second language articulation in a patient with foreign accent syndrome. NeuroReport, 15(14), 2267-70.

Baumann, S. (2006). Information Structure and Prosody: Linguistic Categories for Spoken Language Annotation. In S. Sudhoff, D. Lenertová, R. Meyer, S. Pappert, P. Augurzky, I. Mleinek, N. Richter \& J. Schließer (eds.), Methods in Empirical Prosody Research (pp. 153-180). Berlin, New York: De Gruyter.

Berthier, M. L., Ruiz, A., Massone, M. I., Starkstein, S. E. \& Leiguarda, R. C. (1991). Foreign accent syndrome: behavioural and anatomical findings in recovered and non-recovered patients. Aphasiology, 5(2), 129-147.

Blumstein, S. E., Alexander, M. P., Ryalls, J. H., Katz, W. \& Dworetzky, B. (1987). On the Nature of the Foreign Accent Syndrome: A Case Study. Brain and Language, 31, 215244.

Boersma, P. \& Weenink, D. (1992-2011). Praat - doing phonetics by computer. Version 5.0.11 [www.praat.org].

Chafe, W. (1994). Discourse, Consciousness and Time. Chicago: University of Chicago Press. 
Coelho, C. A. \& Robb, M. P. (2001). Acoustic Analysis of Foreign Accent Syndrome: An examination of Three Explanatory Models. Journal of Medical Speech-Language Pathology, 9(4), 227-242.

Cruttenden, A. (2006). The de-accenting of given information: A cognitive universal? In G. Bernini (ed.), The Pragmatic Organisation of Discourse (pp. 311- 355). The Hague: Mouton de Gruyter.

Danly, M. \& Shapiro, B. E. (1982). Speech prosody in Broca's aphasia. Brain and Language, $16,171-190$.

Fridriksson, J., Ryalls, J., Rorden, C., Morgan, P. S., George, M. S. \& Baylis, G. C. (2005). Brain damage and cortical compensation in foreign accent syndrome. Neurocase, 11(5), 319-324.

Grabe, E., Post, B. \& Nolan, F. (2001). Modelling intonational variation in English: the IViE system. In S. Puppel \& G. Demenko (eds.), Proceedings of Prosody 2000, Adam Mickiewicz University, Poznan, Poland.

Graff-Radford, N. R., Cooper, W. E., Colsher, P. L. \& Damasio, A. R. (1986). An Unlearned Foreign "Accent" in a Patient with Aphasia. Brain and Language, 28, 86-94.

Gurd, J., Bessell, N. J., Bladon, R. A. W. \& Bamford, J. M. (1988). A case of foreign accent syndrome, with follow-up clinical, neuropsychological and phonetic descriptions. Neuropsychologia, 26(2), 237-251.

Gussenhoven, C. (2004). The Phonology of Tone and Intonation. Cambridge: Cambridge University Press.

Haley, K. L., Roth, H. L., Helm-Estabrooks, N. \& Thiessen, A. (2010). Foreign accent syndrome due to conversion disorder: Phonetic analyses and clinical course. Journal of Neurolinguistics, 23, 28-43. 
Halliday, M. (1967). Notes on Transitivity and Theme in English, Part 2. Journal of Linguistics, 3, 199-244.

Ingram, J. C. L., McCormack, P. F. \& Kennedy, M. (1992). Phonetic analysis of a case of foreign accent syndrome. Journal of Phonetics, 20, 457-474.

Kanjee, R., Watter, S., Sévigny, A. \& Humphreys, K. R. (2010). A case of foreign accent syndrome: Acoustic analyses and an empirical test of accent perception. Journal of Neurolinguistics, 23, 580-598.

Katz, W. F., Garst, D. M. \& Levitt, J. (2008). The role of prosody in a case of foreign accent syndrome (FAS). Clinical Linguistics and Phonetics, 22(7), 537-566.

Kent, R. D. and J. C. Rosenbek (1982). Prosodic Disturbance and Neurologic Lesion. Brain and Language, 15(2), 259-291.

Kent, R. D. and J. C. Rosenbek (1983). Acoustic Patterns of Apraxia of Speech. Journal of Speech and Hearing Research, 26(2), 231-249.

Kurowski, K. M., Blumstein, S. E. \& Alexander, M. T. (1996). The Foreign Accent Syndrome: A Reconsideration. Brain and Language, 54, 1-25.

Kuschmann, A. (2011). The dimensions of intonation in neurogenic FAS: A typological perspective. Doctoral dissertation. University of Strathclyde.

Ladd, D. R. (1996). Intonational phonology. Cambridge: Cambridge University Press.

Mariën, P., Verhoeven, J., Engelborghs, S., Rooker, S., Pickut, B. A. \& De Deyn, P. P. (2006). A role for the cerebellum in motor speech planning: evidence from foreign accent syndrome. Clinical Neurology and Neurosurgery, 108, 518-522.

Mariën, P., Verhoeven, J., Wackenier, P., Engelborghs, S. \& De Deyn, P. P. (2009). Foreign accent syndrome as a developmental motor speech disorder. Cortex, 45, 870-878. 
Mennen, I., Schaeffler, F., Watt, N. \& Miller, N. (2008). An autosegmental-metrical investigation of intonation in people with Parkinson's Disease. Asia Pacific Journal of Speech, Language, and Hearing, 11(4), 205-219.

Miller, N., Lowit, A. \& O’Sullivan, H. (2006). What makes acquired foreign accent syndrome foreign? Journal of Neurolinguistics, 19, 385-409.

Moen, I. (2006). Analysis of a case of the foreign accent syndrome in terms of the framework of gestural phonology. Journal of Neurolinguistics, 19, 410-423.

Monrad-Krohn, G. H. (1947). Dysprosody or altered 'melody of language'. Brain and Language, 70, 405-415.

Moonis, M., Swearer, J. M., Blumstein, S., Kurowski, K., Licho, R., Kramer, P., Mitchell, A., Osgood, D.L. \& Drachman, D. A. (1996). Foreign Accent Syndrome Following a Closed Head Injury: Perfusion Deficit on Single Photon Emission Tomography with Normal Magnetic Resonance Imaging. Neuropsychiatry, Neuropsychology, and Behavioural Disorders, 9, 272-279.

Nolan, F. (2003). Intonational equivalence: an experimental evaluation of pitch scales. In D. Recasens, M. J. Solé \& J. Romero (eds.), Proceedings of the XVth International Congress of Phonetic Sciences (pp. 771-774). Barcelona, Spain.

Perkins, R. A., Ryalls, J. H., Carson, C. K. \& Whiteside, J. D. (2010). Acoustic analyses of two recovered cases of foreign accent syndrome. Aphasiology, 24(10), 1132-1154.

Pierrehumbert, J. (1980). The phonology and phonetics of English intonation. Doctoral dissertation, MIT, Cambridge, MA: MIT Press.

Pitrelli, J., Beckman, M. E. \& Hirschberg, J. (1994). Evaluation of Prosodic Transcription Labelling Reliability in the ToBI framework. Proceedings of the 1994 International Conference on Spoken Language Processing (pp. 123-126).Yokohama, Japan. 
Poulin, S., Macoir, J., Paquet, N., Fossard, M. \& Gagnon, L. (2007). Psychogenic or neurogenic origin of agrammatism and foreign accent syndrome in a bipolar patient: a case report. Annals of General Psychiatry, 6(1).

Reetz, H. (1999). Artikulatorische und akustische Phonetik. Trier: Wissenschaftlicher Verlag Trier.

Reeves, R. R., Burke, R. S. \& Parker, J. D. (2007). Characteristics of psychotic patients with foreign accent syndrome. Journal of Neuropsychiatry and Clinical Neurosciences, 19(1), 70-76.

Ryalls, J. (1982). Intonation in Broca's aphasia. Neuropsychologia, 20, 355-360.

Ryalls, J. \& Whiteside, J. (2006). An atypical case of Foreign Accent Syndrome. Clinical Linguistics and Phonetics, 20(2-3), 157-162.

Scott, S. K., Clegg, F., Rudge, P. \& Burgess, P. (2006). Foreign accent syndrome, speech rhythm and the functional neuroanatomy of speech production. Journal of Neurolinguistics, 19, 370-384.

Terken, J. \& Hirschberg, J. (1994). Deaccentuation of words prepresenting 'given' information: effects of persistence of grammatical function and surface position. Language and Speech, 37(2), 125-145.

Varley, R. \& Whiteside, S. (2001). What is the underlying impairment in acquired apraxia of speech? Aphasiology, 15(1), 39-49.

Verhoeven, J. \& Mariën, P. (2002). Prosodic Characteristics of a Case of Foreign Accent Syndrome. Antwerp Papers in Linguistics, 100, 130-148.

Verhoeven, J. \& Mariën, P. (2004). Prosody and Foreign Accent Syndrome: a Comparison of Pre- and Post-stroke Speech. Proceedings of the Speech Prosody Conference 2004 (pp. 123-126). Nara, Japan. 
Verhoeven, J. \& Mariën, P. (2010). Neurogenic foreign accent syndrome: Articulatory setting, segments and prosody in a Dutch speaker. Journal of Neurolinguistics, 23(6), 599-614.

Verhoeven, J., Mariën, P., Engelborghs, S., D’Haenen, H. \& De Deyn, P. P. (2005). A foreign speech accent in a case of conversion disorder. Behavioural Neurology, 16, 225-232.

Wendt, B., Bose, I., Scheich, H. \& Ackermann, H. (2007). Speech rhythm of a woman with foreign accent syndrome (FAS). In J. Trouvain \& W. J. Barry (eds.), Proceedings of the XVIth International Congress of Phonetic Sciences (pp. 2009- 1212). Saarbrücken, Germany.

Whitaker, H. (1982). Levels of impairment in disorders of speech. In R. N. Malatesha \& L. C. Hartlage (eds.), Neuropsychology and Cognition (Volume 1) (pp. 168-207). The Hague: Martinus Nijhoff Publishers.

Whiteside, S. \& Varley, R. (1998). A reconceptualisation of apraxia of speech: A synthesis of evidence. Cortex, 34, 221-231.

Ziegler, W. (2002). Task-related factors in oral motor control: speech and oral diadochokinesis in dysarthria and apraxia of speech. Brain and Language, 80(3), 556575. 


\section{Figure captions}

Figure 1: Pitch accent inventory per speaker group in $\%$

Figure 2: Boundary tone inventory per speaker group in \%

Figure 3: Accentuation patterns for new and post-focally given information per speaker in \%

Figure 4: Model of intonation realisation in FAS 